\title{
First report of CART treatment in AL amyloidosis and relapsed/refractory multiple myeloma
}

\begin{abstract}
Aina Oliver-Caldes (D , ,', Raquel Jiménez, ${ }^{1,2,3}$ Marta Español-Rego,,5 Maria Teresa Cibeira, ${ }^{1,2}$ Valentín Ortiz-Maldonado (1) , ${ }^{1}$ Luis F Quintana, ${ }^{2,5,6}$ Paola Castillo, ${ }^{2,5,7}$ Francesca Guijarro, ${ }^{8}$ Natalia Tovar, ${ }^{1,2,3}$ Mercedes Montoro, ${ }^{1}$ Daniel Benitez-Ribas, ${ }^{4,5}$ Alex Bataller (D) ,' E Azucena González-Navarro,, ${ }^{4,5}$ Joan Cid, ${ }^{9}$ Miquel Lozano, ${ }^{9}$ Lorena Perez-Amill, ${ }^{5}$ Beatriz Martin-Antonio (D) , ${ }^{10}$ Mari-Pau Mena, ${ }^{5}$ David F Moreno, ${ }^{1,2}$ Luis Gerardo Rodríguez-Lobato, ${ }^{1,2}$ Josep Maria Campistol, ${ }^{5,6}$ Gonzalo Calvo, ${ }^{11}$ Joan Bladé, ${ }^{1,2}$ Laura Rosiñol, ${ }^{1,2}$ Manel Juan (D) , ${ }^{4,5}$ Mariona Pascal, ${ }^{4}$ Alvaro Urbano-Ispizua, ${ }^{1}$ Carlos Fernández de Larrea (10) ${ }^{1,2}$
\end{abstract}

To cite: Oliver-Caldes A, Jiménez R, Español-Rego M, et al. First report of CART treatment in $\mathrm{AL}$ amyloidosis and relapsed/refractory multiple myeloma. Journal for ImmunoTherapy of Cancer 2021;9:e003783. doi:10.1136/ jitc-2021-003783

Accepted 14 November 2021

Check for updates

(C) Author(s) (or their employer(s)) 2021. Re-use permitted under CC BY-NC. No commercial re-use. See rights and permissions. Published by BMJ.

For numbered affiliations see end of article.

Correspondence to Dr Carlos Fernández de Larrea; CFERNAN1@clinic.cat

\section{ABSTRACT}

Multiple myeloma (MM) remains incurable despite the number of novel therapies that have become available in recent years. Occasionally, a patient with MM will develop an amyloid light-chain (AL) amyloidosis with organ dysfunction. Chimeric antigen receptor T-cell (CART) therapy has become a promising approach in treating hematological malignancies. Our institution has developed a second-generation B-cell maturation antigen (BCMA)CART which is currently being tested in a clinical trial for relapsed/refractory MM.

We present the first reported case, to our knowledge, of a patient with AL amyloidosis and renal involvement in the course of an MM, successfully treated with CART therapy targeting BCMA. The patient received a fractioned dose of $3 \times 10^{6} / \mathrm{kg}$ BCMA-CARTs after lymphodepletion. At 3 months from infusion, the patient had already obtained a deep hematological response with negative measurable residual disease by flow cytometry in the bone marrow. After 12 months, the patient remains in hematological stringent complete remission and has achieved an organ renal response with a decrease of $70 \%$ of proteinuria. This case suggests that concomitant AL amyloidosis in the setting of MM can benefit from CART therapy, even in patients in which predominant symptoms at the time of treating are caused by $\mathrm{AL}$ amyloidosis.

\section{INSIGHTS}

Chimeric antigen receptor T-cell (CART) therapy may be useful in the treatment of selected cases of amyloid light-chain (AL) amyloidosis.

\section{CASE REPORT}

\section{Background}

In recent years, CART therapy has become a groundbreaking approach in the treatment of hematological malignancies. In the field of plasma cell disorders, multiple myeloma (MM) remains an incurable disease to current standard of care therapies, and therefore, several investigational CART constructs are in development. B-cell maturation antigen (BCMA)-targeted CARTs are showing impressive results in phase I/II clinical trials enrolling patients with MM. ${ }^{1}$ However, MM may occasionally evolve to AL amyloidosis, and no data have been published about the potential benefit of CART therapy in these patients, specifically when AL amyloidosis symptoms are predominant. Our institution has developed an academic second-generation humanized 4-1BB-based CART lentivirally transduced on autologous $\mathrm{T}$ cells targeting BCMA, called ARI0002h. ${ }^{2}$ ARI0002h targets BCMA and is currently being tested in the CARTBCMA-HCP-01 clinical trial for patients with relapsed/refractory MM (ClinicalTrials: NCT04309981).

$\mathrm{AL}$ amyloidosis is a plasma cell disorder characterized by the extracellular deposition of monoclonal light chains in different tissues causing organ dysfunction, especially the kidney and the heart. Here we present a patient with MM who developed AL amyloidosis with significant albuminuria due to renal involvement and was successfully treated with the ARI0002h CART.

\section{RESULTS}

A woman in her early 60 s was diagnosed with an IgA-lambda symptomatic MM in 2014 (Revised International Staging System 2). The patient underwent induction treatment 
with six cycles of bortezomib, lenalidomide and dexamethasone (VRD) followed by an autologous stem cell transplantation (ASCT) conditioned with busulfan and melphalan. Afterwards, she completed two consolidation cycles of VRD achieving a stringent complete response (sCR). The patient started maintenance therapy in a clinical trial with lenalidomide, dexamethasone and ixazomib, but presented a serological relapse after 19 cycles. Salvage therapy based on daratumumab, carfilzomib and dexamethasone was then started, achieving a very good partial response (VGPR). Due to gastrointestinal toxicity related to carfilzomib at cycle 14, daratumumab monotherapy was continued. Ten months later, the patient presented with an increase in the serum M-protein $(21 \mathrm{~g} / \mathrm{L})$, kappa/ lambda serum free light chain (sFLC) of $2 / 231 \mathrm{mg} / \mathrm{L}$ and bone marrow (BM) infiltration by $23 \%$ plasma cells (normal fluorescence in situ hybridization-FISH). There was no evidence of extramedullary disease by positron emission tomography-CT and no hypercalcemia, renal failure, anemia and bone fracture signs were found. However, she developed edema and significant nonselective albuminuria (24-hour proteinuria of $2626 \mathrm{mg}$ with urinary M-protein of $307 \mathrm{mg}$ and serum albumin of $28 \mathrm{~g} / \mathrm{L}$ ) with preserved renal function (creatinine $0.6 \mathrm{mg}$ / dL) (table 1). Therefore, a subcutaneous fat aspiration and a renal biopsy were performed showing lambda-type amyloid deposits (figure 1A-F). Diagnosis of systemic AL amyloidosis with renal involvement (revised Mayo stage II) was established. Cardiac involvement was ruled out by biomarkers and imaging. Although the patient had serological progression, at this point renal, AL amyloidosis was the main reason to initiate treatment.

Due to the previous findings and having received prior treatment with proteasome inhibitors (PIs), immunomodulatory drugs (IMIDs) and a CD38-targeted monoclonal antibody, the patient was treated with the ARI0002h BCMA-CART under compassionate use, as AL was an exclusion criterion in the clinical trial. Lymphodepletion was administered in days $-6,-5$ and -4 with fludarabine (total dose $90 \mathrm{mg} / \mathrm{m}^{2}$ ) and cyclophosphamide (total dose $900 \mathrm{mg} / \mathrm{m}^{2}$ ), followed by the infusion of $3 \times 10^{6}$ ARI0002h cells $/ \mathrm{kg}$ in a fractionated manner: $10 \%$ on day $0,30 \%$ on day +2 and the remaining $60 \%$ on day +6 . The patient developed a grade 1 cytokine release syndrome on day +8 , consisting of fever, elevation of acute-phase reactants (ferritin $1388 \mathrm{ng} / \mathrm{mL}$; C reactive protein $3.89 \mathrm{mg} / \mathrm{dL}$, with previous determinations in normal range) without renal toxicity and no isolations in the microbiological tests, completely resolving after 48 hours with no need for tocilizumab or any specific intervention. Treatmentrelated grade 4 neutropenia and grade 3 thrombocytopenia were observed; both improved at day +33 , although asymptomatic cytopenias (grade 2 neutropenia and grade 1 thrombocytopenia) are persistent after 12 months. No evidence of immune effector cell-associated neurotoxicity syndrome was observed.

In the following months, the patient developed two viral complications. A SARS-CoV-2 pneumonia, which required remdesivir and convalescent plasma, but not admission of the patient into intensive care unit. COVID-19 symptoms resolved within the first 3 weeks, but a positive asymptomatic SARS-CoV-2 PCR on nasopharyngeal swab sample persisted for 3 months. The provided treatments completely resolved both complications and the patient developed SARS-CoV-2 antibodies. She is now fully vaccinated. Also, 2 months after infusion of ARI0002h, the patient was diagnosed with a BK virus hemorrhagic cystitis after an onset of urine retention and hematuria with clots and a BK virus measurement on urine of $23 \times 10^{6}$ copies $/ \mathrm{mL}$. A cystoscopy with biopsy also revealed the presence of amyloid in the bladder. The patient was initially treated with clot extraction and bladder irrigation with intermittent resolution of the symptoms, as well as intravenous immunoglobulin. At 4 months from infusion, due to recurring episodes, a bilateral vesical artery embolization was performed, with complete resolution of the complication. Although BK virus was considered the main etiology of this complication, AL amyloidosis vesical involvement may have contributed to the severity of the symptoms. Also, the platelet count decreased from grade 1 to 2 since day +33 to grade 3 after the onset of vesical bleeding. For that reason, platelet transfusions were administered to maintain a platelet count over $50 \times 10^{9} / \mathrm{L}$.

In the first evaluation of response 1 month after CART infusion, the patient had achieved a hematological PR, with suppressed lambda sFLC, a serum M-protein of $4.7 \mathrm{~g} / \mathrm{L}$ and no detectable M-protein in the urine. Clonal plasma cells were absent in a BM aspirate by flow cytometry (sensitivity $10^{-4}$ ). In terms of organ involvement, a renal response was observed with proteinuria decreasing to $1777 \mathrm{mg} / 24$ hours (32\% decrease) (figure 1G).

Three months after ARI0002h administration, serum and urinary M-protein were not detectable by immunofixation, and BM MRD by next-generation flow (NGF) continued to be negative (sensitivity $2.1 \times 10^{-6}$ ). Proteinuria was not evaluable due to the urinary bleeding in the context of the BK virus hemorrhagic cystitis. At the 6 month follow-up, the patient remained in hematological sCR with negative MRD by NGF (sensitivity $2 \times 10^{-6}$ ) and an improved renal response with proteinuria decreasing by $55 \%$ to $1185 \mathrm{mg} / 24$ hours. At the 12-month follow-up, the patient remains in hematological sCR with negative MRD (sensitivity $2 \times 10^{-6}$ ) and in renal response with a proteinuria of $797 \mathrm{mg} / 24$ hours (total decrease of $70 \%$ ) (figure 1G). Hematological and organ response was evaluated according to the staging system proposed by Palladini $e t a .^{3}$

In terms of CART expansion and persistence, the highest peak of expansion was detected at day +21 of CART infusion and the CART was detectable until day +84 (figure 1G). Clonality analysis of FR1 showed a clonal relationship between the original BM plasma cells at diagnosis and at the time of AL amyloidosis. 


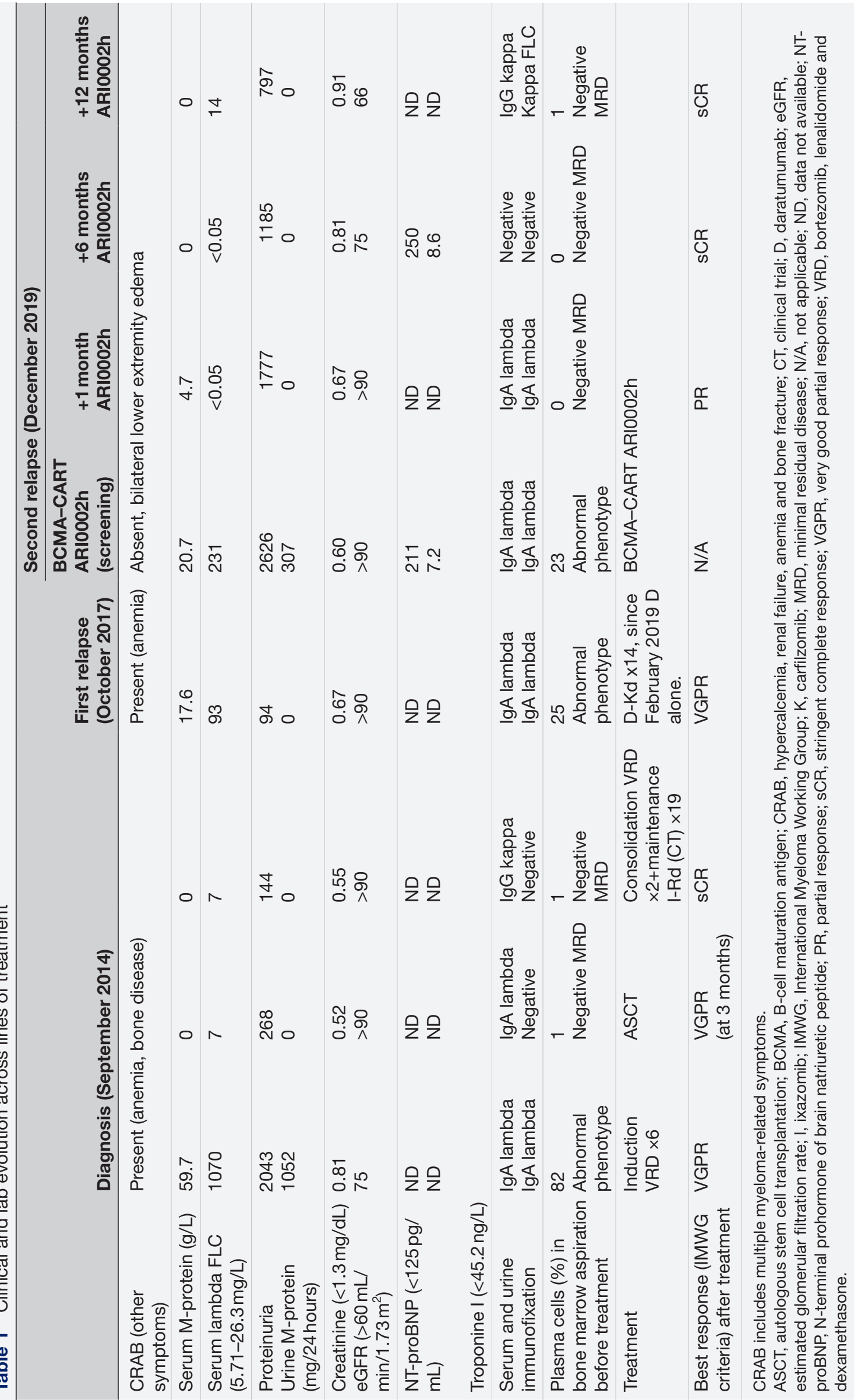




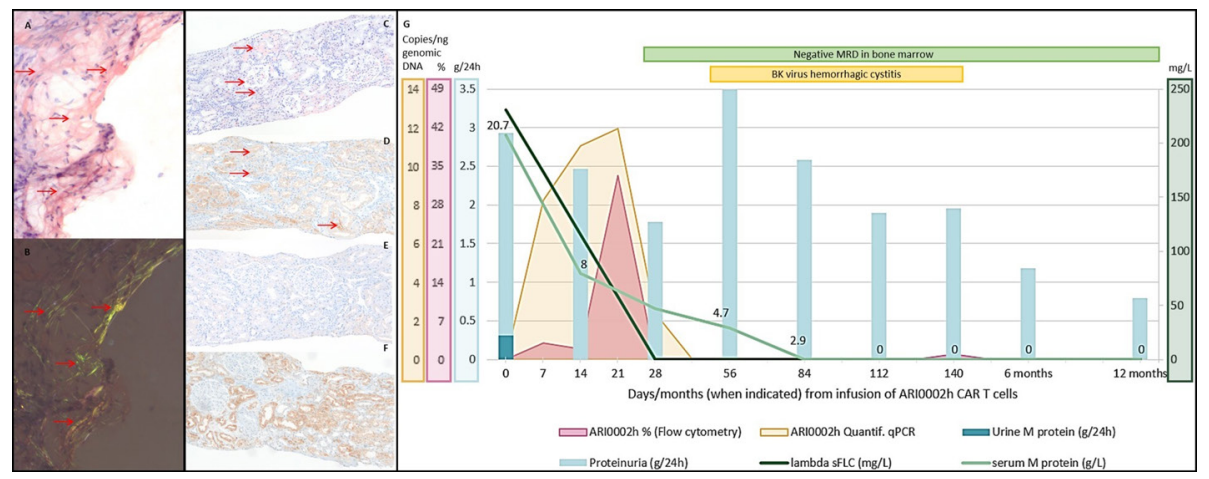

Figure 1 Diagnosis and immunohistochemical typing of amyloidosis. Evolution of clinical and laboratory findings after infusion of ARI0002h. Diagnosis of amyloid deposits in a fine-needle aspiration of subcutaneous fatty tissue: (A) Congo red-stained tissue section, amyloid deposits highlighted with horizontal arrows; (B) Congo red green birefringence, amyloid deposits highlighted with horizontal arrows. Immunohistochemical typing of the renal amyloidosis as AL $\lambda$ type shown on adjacent sections and amyloid deposits highlighted with horizontal arrows: (C) Congo red-stained (D) anti-AL $\lambda$ showing positivity on amyloid deposits; (E) anti-AL $\kappa$ and (F) anti-AA showing negativity on amyloid deposits. Magnification $\times 100$. Amyloid was not evident in the bone marrow section. (G) In the first 6 months after infusion of ARI0002h, a progressive decline in serum Mprotein and lambda sFLC was observed. MRD was negative on day +28 and remains negative at 12 months. A renal response with a $55 \%$ and $70 \%$ decrease in proteinuria was observed at 6 and 12 months, with an intermediate period in which, due to hemorrhagic cystitis, proteinuria is not assessable. Expansion of CARTs by either flow cytometry and qRT-PCR are also represented, with a peak at day +21 . AL, amyloid light-chain; CART, chimeric antigen receptor T cell; MRD, minimal residual disease; sFLC, serum free light chain.

\section{CONCLUSION}

Here, we present the case of a patient with systemic AL amyloidosis, successfully treated with BCMA-targeted CART therapy. Even though this is not a typical case of AL amyloidosis with heart involvement, and that was developed during the course of symptomatic MM, AL was the main reason to initiate treatment since MM-related symptoms were ruled out at that point. To our knowledge, this is the first reported use of CART therapy for this disease. In this patient, the main toxicities were SARS-CoV-2 pneumonia, attributable to the epidemiological situation, and a BK virus hemorrhagic cystitis, a complication that has been reported in patients treated with CD19-targeted CART therapy. ${ }^{4}$

AL amyloidosis in the setting of MM has been reported in $5 \%-7 \%$ of patients with overt organ dysfunction and in up to $10 \%-15 \%$ of a necropsy series of end-stage MM. Desikan et al found amyloid in histological samples of 32 of $84(38 \%)$ minimally treated patients with MM (none or one prior line of treatment) consecutively included in a protocol, although only $8 \%(\mathrm{n}=7)$ had symptomatic organ involvement. ${ }^{5}$

The main purpose of AL amyloidosis treatment is to quickly and fully eliminate the plasma cell clone (hematological response) in order to decrease free light-chain (FLC) deposition on tissues and to improve or reverse organ damage (organ response). Until now, the treatment approach of AL amyloidosis has been extrapolated from the treatment of MM with the use of PIs, IMIDs and high dose melphalan followed by ASCT in eligible patients. ${ }^{6}$ More recently, a phase III trial comparing daratumumab in combination with cyclophosphamide, bortezomib and dexamethasone has shown high rates of hematological and organ response ( $42 \%$ for cardiac response and $54 \%$ for renal response). ${ }^{7}$ These data support the use of daratumumab in AL amyloidosis treatment, similar to its usage in MM. However, the treatment options for patients with refractory disease to both daratumumab and bortezomib are limited. ${ }^{6}$ There are already clinical trials under way that exploit BCMA-targeted therapy in AL amyloidosis (ie, ClinicalTrials: NCT04617925).

Due to this need to achieving fast and deep hematological responses plus the efficacy seen in this patient, authors believe that BCMA-CART therapy should be considered in the future, in patients in which organ involvement is not a limitation itself for CART therapy for reasons of potential toxicity. AL amyloidosis is still an exclusion criterion in our protocol, but a future trial for AL amyloidosis patients is planned.

Reported data regarding BCMA expression on amyloidosis plasma cells show a median membrane BCMA expression of $38.5 \%$ in 12 BM samples assessed by flow cytometry after CD138 isolation ${ }^{8}$ and $80 \%$ (range 20\%-100\%) in clonal plasma cells of $28 \mathrm{BM}$ biopsy samples assessed by immunohistochemistry, ${ }^{9}$ suggesting that BCMA may be an appropriate target in AL amyloidosis.

In our patient, BCMA expression on BM plasma cells obtained before CART infusion was 22\% (Phycoerythrin (PE) antihuman CD269 antibody, BioLegend ${ }^{\circledR}$ ). Recent results from BCMA-CART trials in MM suggest that BCMA expression on MM plasma cells is not a predictor of response, ${ }^{10}$ and that could explain the lack of association between the relatively low BCMA expression of our patient and her favorable clinical outcome. A very profound response with a difference of less than 20 or even $10 \mathrm{mg} / \mathrm{L}$ between involved and uninvolved sFLC, as achieved by our patient, has been postulated as a 
predictive factor of organ response and survival in $\mathrm{AL}$ amyloidosis. ${ }^{11}$

This case suggests that concomitant AL amyloidosis in the setting of MM may benefit from CART therapy, even in patients in which predominant symptoms are caused by AL amyloidosis. However, organ involvement of some patients with AL amyloidosis, particularly those with cardiac compromise, may make most of them not eligible; there are important side effects, including cytopenias and immunosupression. Renal involvement by AL amyloidosis was the main reason to start therapy. Put together with the high efficacy of ARI0002h in our patient, we consider that CART cells might be a promising therapeutic strategy for carefully selected patients with this disease.

\section{Author affiliations}

${ }^{1}$ Hematology Department, Institut d'Investigacions Biomèdiques August Pi i Sunyer (IDIBAPS), Hospital Clinic de Barcelona, Barcelona, Spain

${ }^{2}$ Amyloidosis and Multiple Myeloma Unit, Hospital Clinic de Barcelona, Barcelona, Spain

${ }^{3}$ Clinical Trial Unit, Hospital Clinic de Barcelona, Barcelona, Spain

${ }^{4}$ Immunology Department, Hospital Clinic de Barcelona, Barcelona, Spain

${ }^{5}$ Institut d'Investigacions Biomèdiques August Pi i Sunyer (IDIBAPS), Hospital Clinic

de Barcelona, Barcelona, Spain

${ }^{6}$ Glomerular Disease Unit (CSUR), Nephrology Department, Hospital Clinic de

Barcelona, Barcelona, Spain

${ }^{7}$ Pathology Department, Hospital Clinic de Barcelona, Barcelona, Spain

${ }^{8}$ Hematopathology Unit, Hospital Clinic de Barcelona, Barcelona, Spain

${ }^{9}$ Apheresis Unit, Hospital Clinic de Barcelona, Barcelona, Spain

${ }^{10}$ Instituto de Investigación Sanitaria, Fundación Jiménez Díaz, Madrid, Spain

${ }^{11}$ Clinical Pharmacology Department, Hospital Clinic de Barcelona, Barcelona, Spain

Correction notice This article has been corrected since it was first published online. The funding statement has been updated to include the statement "This study has been funded by Instituto de Salud Carlos III through the project "ICl19/00025" (Co-funded by European Regional Development Fund "A way to make Europe")".

\section{Twitter Aina Oliver-Caldes @ainitaoliver}

Acknowledgements The authors thank all the staff members of the hematology, immunology, pathology, nephrology and clinical pharmacology departments, the Amyloidosis and Myeloma and the Apheresis Units of Hospital Clinic of Barcelona involved in the treatment of the patient, as well as all the personnel involved in the preclinical development of this institutional chimeric antigen receptor T-cell construct. The authors also thank Anthony Battram for his help with manuscript editing.

Contributors AO-C, RJ, ME-R, MTC, VO-M, LFQ, JMC, NT and CFdL participated in the conception and design of the work. AO-C, LPA, BM-A and AU-I participated in the writing of the manuscript. PC, DB-R and FG provided data and figures. MM, $\mathrm{AB}, \mathrm{EAG}-\mathrm{N}, \mathrm{JMC}$ and ML collaborated with data collection. DFM, LGR-L, MER, GC, $\mathrm{JB}$ and MP performed data analysis and interpretation. LR, MTC, MJ, AU-I and CFdL revised the article. All the authors gave the final approval of the version to be published.

Funding This study has been funded by Instituto de Salud Carlos III through the project "ICl19/00025" (Co-funded by European Regional Development Fund "A way to make Europe"), Spanish Ministry of Health (FIS PI18/00775, PI19/00669, ICl19/00025 and complementary grant for CONCORD-023), Fondo Europeo de Desarrollo Regional and 2017SGR00792 (AGAUR, Generalitat de Catalunya) and 'La Caixa' Foundation (CP042702). A0-C received funding from the resident grant 'Ajut Clínic-La Pedrera' 2019, granted by Hospital Clínic de Barcelona. As a BITRECS fellow, LGR-L has received funding from the European Union's Horizon 2020 research and innovation program under the Marie Sklodowska-Curie grant agreement ID 754550 and from 'La Caixa' Foundation.

Competing interests None declared.

Patient consent for publication Not applicable.

Ethics approval The patient underwent treatment with ARI0002h as a compassionate use (amyloidosis was an exclusion criterion in the clinical trial) after approval by the local Health Care Ethics Committee and the Spanish Agency for Medications and Healthcare Products. An informed consent form was obtained. Patient medical record was collected for analysis.

Provenance and peer review Not commissioned; externally peer reviewed.

Open access This is an open access article distributed in accordance with the Creative Commons Attribution Non Commercial (CC BY-NC 4.0) license, which permits others to distribute, remix, adapt, build upon this work non-commercially, and license their derivative works on different terms, provided the original work is properly cited, appropriate credit is given, any changes made indicated, and the use is non-commercial. See http://creativecommons.org/licenses/by-nc/4.0/.

\section{ORCID iDs}

Aina Oliver-Caldes http://orcid.org/0000-0002-7921-5420

Valentín Ortiz-Maldonado http://orcid.org/0000-0003-4699-6862

Alex Bataller http://orcid.org/0000-0002-6085-2745

Beatriz Martin-Antonio http://orcid.org/0000-0003-0612-2693

Manel Juan http://orcid.org/0000-0002-3064-1648

Carlos Fernández de Larrea http://orcid.org/0000-0003-4930-9255

\section{REFERENCES}

1 Shah UA, Smith EL. Multiple myeloma, targeting B-cell maturation antigen with chimeric antigen receptor T-cells. Cancer $J$ 2019;25:208-16.

2 Perez-Amill L, Suñe G, Antoñana-Vildosola A, et al. Preclinical development of a humanized chimeric antigen receptor against $\mathrm{B}$ cell maturation antigen for multiple myeloma. Haematologica 2021;106:173-84.

3 Palladini G, Hegenbart U, Milani P, et al. A staging system for renal outcome and early markers of renal response to chemotherapy in $A L$ amyloidosis. Blood 2014;124:2325-32.

4 Khan AM, Ajmal Z, Tuz Zahra F, et al. Hemorrhagic cystitis secondary to adenovirus and BK virus infection in a diffuse large B-cell lymphoma patient with recent CAR T-cell therapy. Case Rep Hematol 2020;2020:6621967:1-4.

5 Desikan KR, Dhodapkar MV, Hough A, et al. Incidence and impact of light chain associated (al) amyloidosis on the prognosis of patients with multiple myeloma treated with autologous transplantation. Leuk Lymphoma 1997;27:315-9.

6 Palladini G, Milani P, Merlini G. Management of AL amyloidosis in 2020. Blood 2020:136:2620-7.

7 Kastritis E, Palladini G, Minnema MC, et al. Daratumumab-based treatment for immunoglobulin light-chain amyloidosis. N Engl J Med 2021:385:46-58.

8 Godara A, Zhou P, Kugelmass A, et al. Presence of soluble and cellsurface B-cell maturation antigen in systemic light-chain amyloidosis and its modulation by gamma-secretase inhibition. Am J Hematol 2020;95:E110-3.

9 Bal S, Sigler A, Chan A, et al. First description of B cell maturation antigen expression in light chain amyloidosis. Blood 2019;134:5452.

10 Raje N, Berdeja J, Lin Y, et al. Anti-BCMA CAR T-Cell Therapy bb2121 in Relapsed or Refractory Multiple Myeloma. N Engl J Med 2019;380:1726-37.

11 Milani P, Basset M, Nuvolone M, et al. Indicators of profound hematologic response in $\mathrm{AL}$ amyloidosis: complete response remains the goal of therapy. Blood Cancer J 2020;10:90. 
Correction: First report of CART treatment in AL

Amyloidosis and relapsed/refractory multiple myeloma

Oliver-Caldes A, Jiménez R, Español-Rego M, et al. First report of CART treatment in AL amyloidosis and relapsed/refractory multiple myeloma. J Immunother Cancer 2021;9:e003783. doi: 10.1136/jitc-2021-003783

The funding statement has been updated to include the statement "This study has been funded by Instituto de Salud Carlos III through the project "ICI19/00025" (Co-funded by European Regional Development Fund "A way to make Europe")".

(C) Author(s) (or their employer(s)) 2022. No commercial re-use. See rights and permissions. Published by BMJ.

J Immunother Cancer 2022;10:e003783corr1. doi:10.1136/jitc-2021-003783corr1

(D) Check for updates 